\title{
Transformation of Chilli Pepper (Capsicum frutescens) with a Phenylalanine Ammonia-Lyase Gene
}

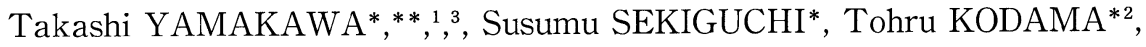 \\ Steven M. SMITH**, and Michael M. YEOMAN** \\ * Department of Biotechnology, The University of Tokyo, Bunkyo-ku, Tokyo 113-8657, Japan \\ ** Institute of Cell and Molecular Biology, The University of Edinburgh, The King's \\ Buildings, Mayfield Road, Edinburgh EH9 3JH, UK
}

Received 24 August 1998; accepted 17 September 1998

\begin{abstract}
Hypocotyl explants of chilli pepper (Capsicum frutescens) were inoculated with Agrobacterium rhizogenes strain A13 harbouring the binary vector pBI121, which contains the CaMV 35S promoter linked to the GUS reporter gene. After 4 weeks of culture, the hairy roots produced were transferred onto Schenk and Hildebrandt ( $\mathrm{SH}$ ) medium supplemented with $250 \mathrm{mg} l^{-1}$ Cefotaxime. Subsequently they were selected in liquid SH medium containing $20 \mathrm{mg} l^{-1}$ kanamycin. When treated with $\mathrm{X}$-glucuronide, vascular and root tip tissues were predominantly stained. A phenylalanine ammonia-lyase (PAL) cDNA from parsley (Petroselinum) was subsequently linked to the 35S promoter and transferred into hairy roots using the same A. rhizogenes strain. Successful transformation was confirmed by Southern hybridisation. Hairy roots containing the PAL transgene showed different PAL activity, slow growth and altered morphology.
\end{abstract}

\section{Introduction}

Capsaicin and its dihydro derivative are responsible for the hot taste of peppers (Capsicum frutescens L.) and have therefore received much attention. The relationship between the synthesis and accumulation of capsaicin has been studied in plants and cultured cells of $C$. frutescens [1-4]. Future progress in understanding the regulation of capsaicin accumulation could be significantly accelerated by the application of genetic manipulation techniques. Furthermore, the genetic transformation of chilli pepper and its relatives could be employed in pepper breeding programmes. Agrobacterium-mediated transformation of bell pepper (Capsicum annuum L.) has been reported[5]. In this case Agrobacterium tumefaciens strains C58 and A281 were used to induce tumour formation on cotyledon, hypocotyl and leaf explants, and the introduction of a GUS reporter gene was demonstrated by histochemical staining with $\mathrm{X}$-glucuronide. The aim of the work presented here was to develop a genetic transformation protocol for $C$. frutescens and to investigate the potential for genetic manipulation of the phenylpropanoid pathway leading to defense molecules, lignin, pigments and flavours including

1 Present address: Department of Global Agricultural Sciences, The University of Tokyo, Bunkyo-ku, Tokyo 113-8657, Japan

Present address: Faculty of Textile Science and Technology,

Shinshu University, Ueda, Nagano 386-8567, Japan

3 To whom correspondence should be addressed. capsaicin.

\section{Materials and Methods}

\section{1 Plant material}

Chilli pepper (Capsicum frutescens cv. Cayenne) seeds were surface-sterilized with ethanol for a few seconds followed by $10 \%$ sodium hypochloride for 15 min. After three times rinse in sterile water, seeds were germinated at $25^{\circ} \mathrm{C}$ under continuous light on Schenk and Hildebrandt (SH) medium[6] with $1 \%$ agar.

\section{2 Agrobacterium strains and plasmids}

The $A$. rhizogenes strains used for transformation of chilli pepper were A5, A13 (supplied by Professor H. Kamada, The University of Tsukuba, Japan), and 1610[7]. A binary vector pBI121 carried the CaMV35S promoter-GUS gene fusion and the neomycin phosphotransferase gene as a selectable marker[8]. A chimeric plasmid pBI12PPAL was constructed with pBI121 by substituting the GUS gene for a full length parsley PAL-2 cDNA (Fig. 1). Parsley PAL-2 cDNA was a courtesy gift from Professor K. Hahlbrock (Max-Plank Institute, Cologne Germany) [9].

The Agrobacterium rhizogenes strains carrying the pBI121-derived vector were produced by a triparental mating with Escherichia coli HB101 that harboured pRK2013 and E. coli JM101 that harboured the plas- 


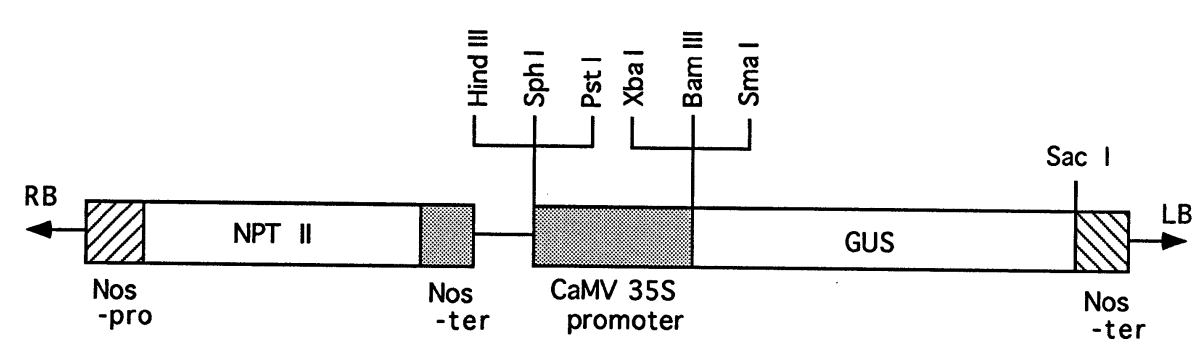

$\mathrm{pBI} 121$

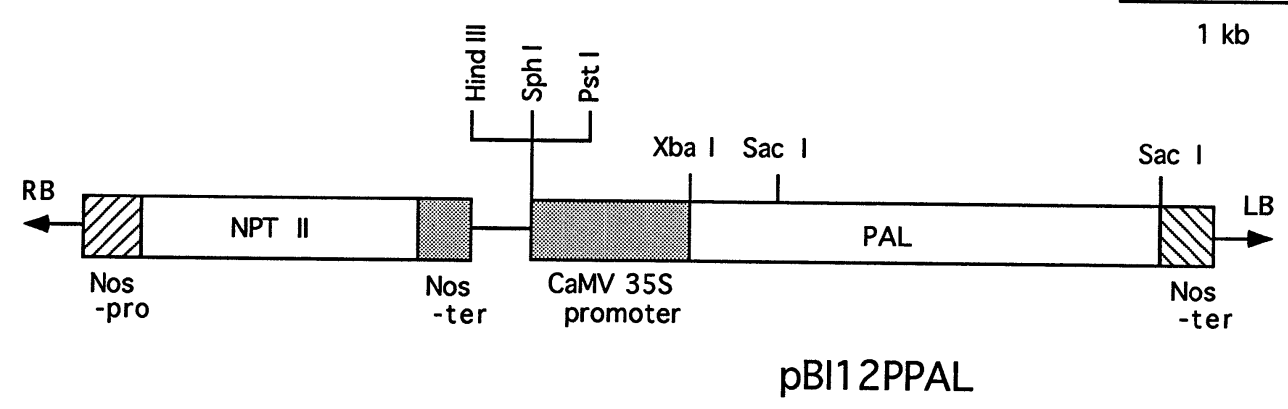

Fig. 1 T-DNA maps of the binary vector plasmid pBI121 and pBI12PPAL.

mid. After single colony isolations, E. coli and $A$. rhizogenes strains carring the constructed plasmid were cultured overnight with selectable antibiotics, then immediately stored at $-80^{\circ} \mathrm{C}$ in $35 \%(\mathrm{v} / \mathrm{v})$ glycerol. This minimised the occurance of deletion derivatives of the plasmid which may otherwise have been selected due to toxicity of cinnamic acid produced following expression of the PAL cDNA in bacteria.

\section{3 Transformation}

A. rhizogenes cultures were grown overnight with vigorous agitation at $25^{\circ} \mathrm{C}$ in $\mathrm{LB}$ medium containing the selective antibiotics appropriate for each plasmid. Fifty microlitre of these cultures were plated on LB agar medium overnight. The $1.5 \mathrm{~cm}$ explants from hypocotyls of chilli pepper were inoculated with $A$. rhizogenes and were cultured at $25^{\circ} \mathrm{C}$ under continuous light on semi-solid SH medium for 4 weeks. The hairy roots which grew were transferred individually onto SH medium supplemented with $250 \mathrm{mg} l^{-1}$ Cefotaxime. Subsequently they were selected in liquid SH medium containing $20 \mathrm{mg} l^{-1}$ kanamycin.

\section{4 GUS histochemical assay}

Hairy roots were subjected to X-glucuronide treatment according to the CLONTECH (Palo Alto, California, USA) manual. GUS-expressing cells were detected microscopically by the distinct blue colour that developed as a result of enzymatic cleavage of $\mathrm{X}$-glucuronide.

\section{5 DNA extraction, polymerase chain reaction $(P C R)$ and Southern blot analysis}

DNA extraction employed the SDS method[10]. PCR was carried out using 20-mer oligonucleotides (for NPT II gene detection, 5'-CCTTGCAGTCACCTCGTAAA- $3^{\prime}$ and $5^{\prime}$-CGCAAGACCGGCAACAGGAT- $3^{\prime}$ ) and 21-mer oligonucleotides (for GUS gene detection, $5^{\prime}$-GGTGGGAAAGCGCGTTACAAG - 3' and $5^{\prime}$-GTTTACGCGTTGCTTCCGCCA - 3') [11] with the following cycle parameters: $30 \mathrm{sec}$ at $94^{\circ} \mathrm{C}$, $1 \mathrm{~min}$ at $55^{\circ} \mathrm{C}, 2 \mathrm{~min}$ at $72^{\circ} \mathrm{C}$. The first cycle used an additional $5 \mathrm{~min}$ melt at $95^{\circ} \mathrm{C}$. For Southern hybridization, approximately $4 \mu \mathrm{g}$ total DNA digested with the restriction enzyme was transferred to a Hybond$\mathrm{N}$ nylon membrane. Blots were hybridized to $2 \mathrm{~kb}$ pBI12PPAL Sac I DNA-fragment labeled with digoxigenine (DIG; Boehringer Mannheim). Conditions for Southern analysis followed those described by the DIG DNA labelling kit.

\section{6 Determination of PAL (E. C. 4. 3. 1. 5.) activity}

PAL activity was measured spectrophotometrically according to the method of Bolwell et al.[12]. Root tissue was homogenized in borate buffer $\left(0.05 \mathrm{M} \mathrm{Na}_{2}\right.$ $\mathrm{B}_{4} \mathrm{O}_{7}, 0.2 \mathrm{M} \mathrm{H}_{3} \mathrm{BO}_{4}, 0.05 \mathrm{M} \mathrm{NaCl}, \mathrm{pH}$ 8. 8) containing $2 \mathrm{mM}$ mercaptoethanol. The homogenate was centrifuged at $20000 \mathrm{~g}$ for $5 \mathrm{~min}$ and the resulting supernatant was used as enzyme preparation. All steps were performed at $2^{\circ} \mathrm{C}$. Protein determinations were carried out according to the method of Bradford [13]. One unit (kat) of enzyme is that required for the formation of $1 \mathrm{~mol}$ of product in $1 \mathrm{sec}$ under the assay conditions.

\section{7 Time course of growth and PAL activity of hairy roots.}

One gram fresh weight of hairy roots was inoculated in $300 \mathrm{~m} l$ Erlenmeyer's flasks containing $200 \mathrm{~m} l \mathrm{SH}$ liquid medium and cultured on a gyratory shaker at $100 \mathrm{rpm}$ at $25^{\circ} \mathrm{C}$. At each sampling time, all roots 


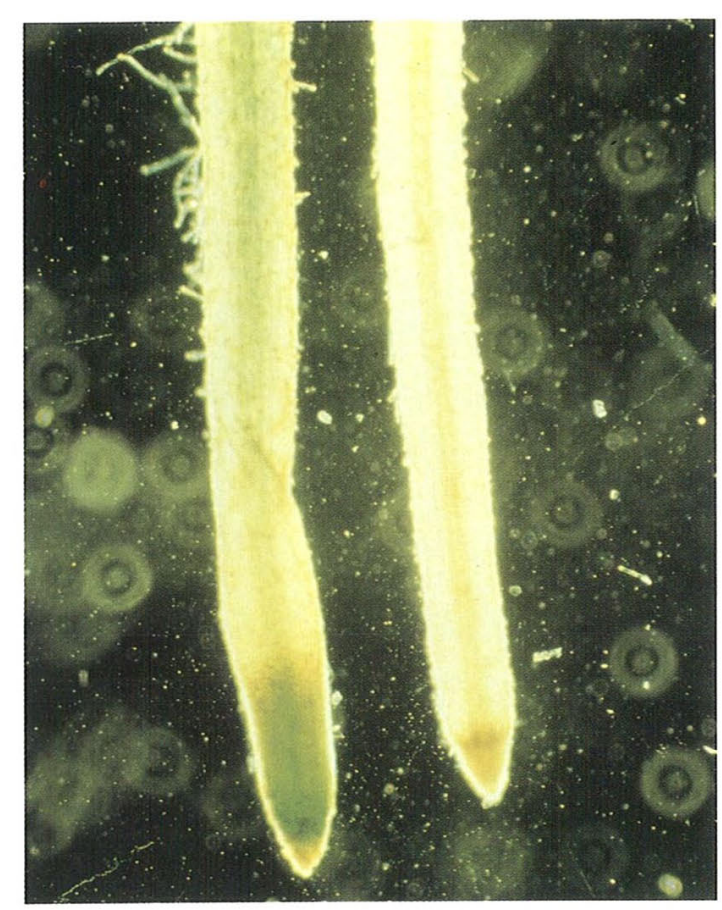

(A)

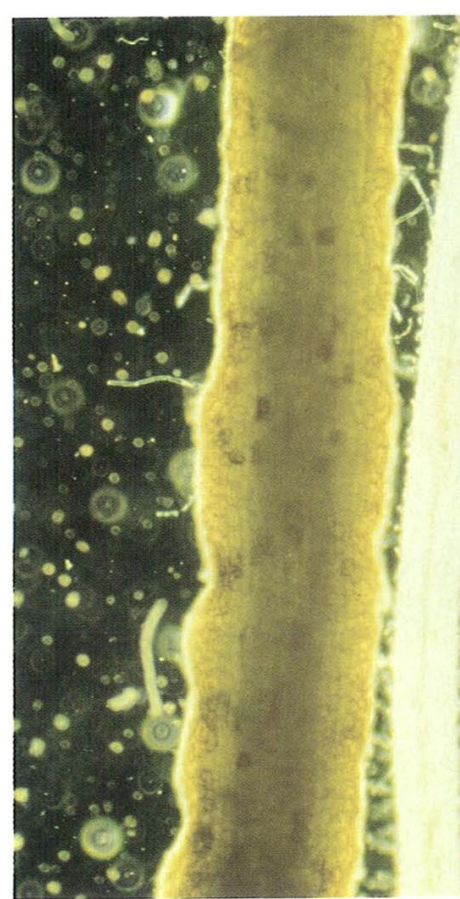

(B)

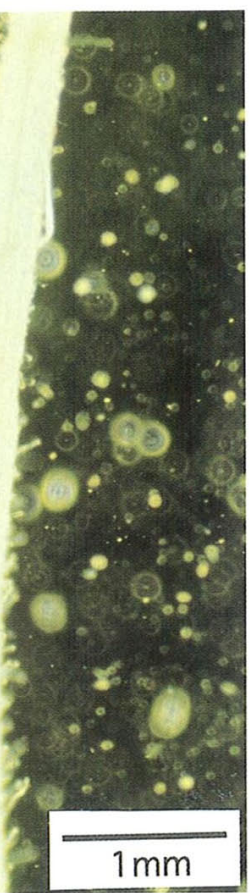

$1 \mathrm{~mm}$

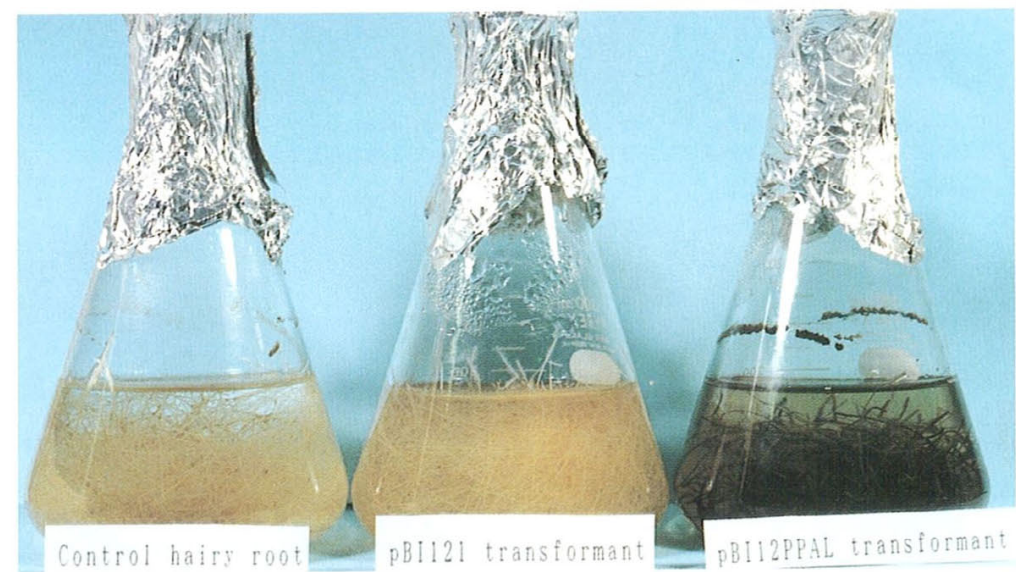

(C)

Fig. 2 (A) Histochemical assay of GUS activity in hairy roots.

Left ; pBI121transformant, Right; Control hairy root

(B) Agrobacterium - mediated transformation of $\mathrm{PAL}$ cDNA.

Left; pBI12PPAL transformant, Right; Control hairy root.

(C) Cultured hairy roots. Culture condition; see the text.

Left; Control hairy root, Middle ; pBI121 transformant,

Right; pBI12PPAL transformant. 
from these flasks were harvested for fresh weight measurements and assay of PAL activities.

\section{Results and Discussion}

\section{1 Transformation}

Three strains of $A$. rhizogenes, (A5, A13 and 1610) each containing $\mathrm{pBI} 121$, were tested for their ability to induce hairy root formation from hypocotyls of $C$. frutescens $\mathrm{cv}$. Cayenne. Each strain was able to induce hairy root formation (not shown). Hypocotyl explants were employed because the subsequent removal of Agrobacterium was easier than with other explants. GUS histochemical staining showed that each Agrobacterium strain was able to transfer the GUS gene successfully. Without kanamycin selection for transgenic hairy roots, the proportions giving blue staining with $\mathrm{X}$-glucuronide were $40 \%$ for $\mathrm{A} 5$, $70 \%$ for A13 and $67 \%$ for 1610 . Strain A13 was used for further studies due to the stability of hairy root growth. Transgenic roots containing the GUS gene could not be selected on medium containing kanamycin within the range 10 to $100 \mathrm{mg} l^{-1}$ because growth of roots both with and without pBI121 was extremely variable. Subsequently hairy roots were transferred to liquid SH medium and shaken at 100 rev $\min ^{-1}$, where about $30 \%$ of roots could be cultured. Addition of $20 \mathrm{mg} \mathrm{l}^{-1}$ kanamycin then allowed reliable selection of transgenic roots, as judged by GUS gene expression.

\section{2 Characterization of GUS gene expression in chilli pepper}

The CaMV 35S promoter is most active in the vascular tissue, as was found with bell pepper leaflike structures[5] and also in the mitotic root tip. This pattern of expression is consistent with previous results for GUS gene expression directed by the CaMV 35S promoter[14]. After one year in culture without kanamycin, GUS activity could still be detected in transgenic roots, indicating that the gene was apparently not susceptible to changes such as methylation, deletion or mutation. These results indicate that the GUS gene was integrated into the genome of hairy roots, and stably transmitted during a prolonged period of culture.

\section{3 Analysis of transformants}

Further transgenic hairy roots were obtained using a modified version of pBI121 in which the GUS coding region was replaced with that from a parsley PAL cDNA, giving pBI12PPAL (Fig. 1). PCR analysis using NPT primers demonstrated that all selected kanamycin-resistant hairy roots, obtained with both pBI121 and pBI12PPAL, contained the NPT II gene

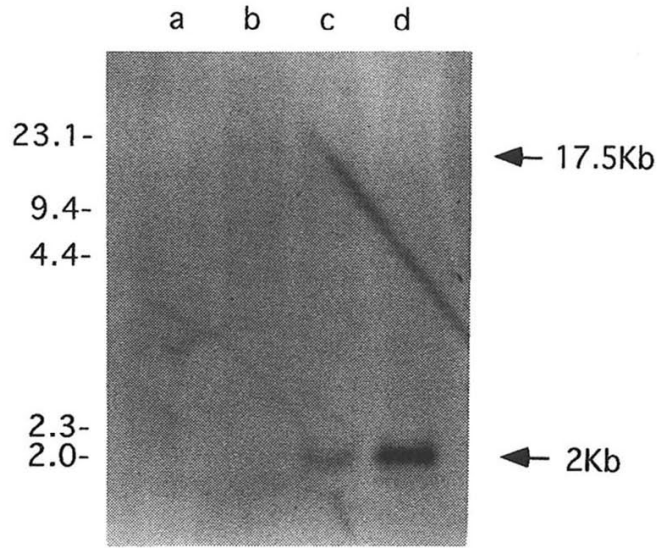

Fig. 3 Surthern blot analysis of pBI121 transformed hairy root I and pBI12PPAL transformed hairy root I with DIG-labeled $2 \mathrm{~kb}$ Sac I frag. ment containing PAL-2 cDNA.

Lane a; Genomic DNA of pBI121 transformed hairy root I digested with Hind III., b; Genomic DNA of pBI12PPAL transformed hairy root I digested with HindIII, c; Genomic DNA of pBI12PPAL transformed hairy root I digested with $S a c$ I d; plasmid pBI12PPAL digested with $\mathrm{Sac}$ I as a positive control.

(Fig. 2). Also, PCR amplification with GUS primers demonstrated the presence of the GUS gene in pBI121 transformants. These PCR products were not detected with DNA from control hairy roots. Since additional non-specific amplification products could also be detected, the presence of the PAL gene in pBI12PPAL transformants was confirmed by Southern blot analysis. Total genomic DNA was extracted from transgenic roots, digested with Sac I and subjected to Southern analysis using a DIG-labelled $2 \mathrm{~kb}$ Sac I fragment from pBI12PPAL as the probe. The results (Fig. 3) showed a single band with the same size as the positive control in pBI12PPAL transformants, but not in pBI121 transformants. When DNA from pBI12PPAL transformant I was digested with HindIII, an enzyme which has only one site in pBI12PPAL, a single $17.5 \mathrm{~kb}$ fragment was obtained, suggesting a single transformation event. Southern analysis of pBI12PPAL transformant II showed double PAL transgene incorporation.

\section{4 Characterization of pBI12PPAL transformant}

The morphology, colour and growth rate of pBI12PPAL transformant I were quite different from pBI121 transformants and control hairy roots (Figs. $2,4)$. Specifically, the roots of pBI12PPAL transformant I grew much slower, had an approximately three-fold greater diameter and dark brown epidermal cells. No such dark brown hairy roots were observed for either control hairy roots or pBI121 transformants, despite examining more than 100 trans- 


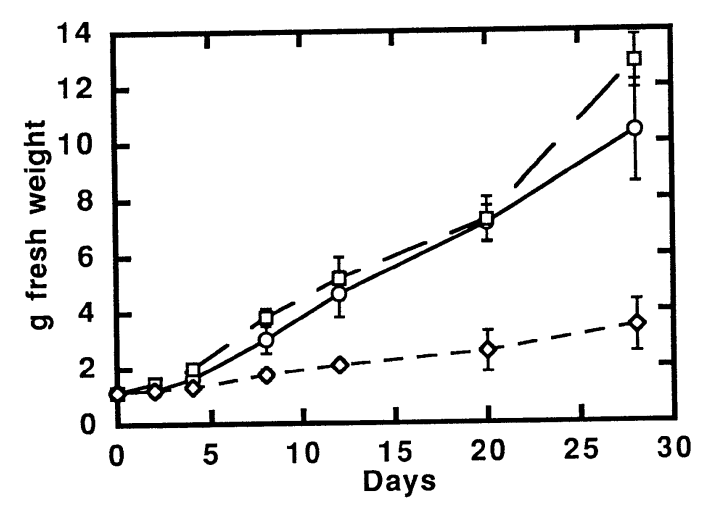

Fig. 4 Growth curves of the hairy roots transformed with pBI121 and pBI12PPAL.

Values represent mean \pm s. e. m. from triplicate flasks. $-\bigcirc^{-}$; Control hairy root, $-\square-$; pBI121 transformed hairy root, $-\diamond-$; pBI12PPAL transformed hairy root.

Table 1. PAL activity and growth of PBI 12 PPAL transformants.

\begin{tabular}{rcccc}
\hline \multicolumn{5}{c}{ PAL activity (n kat/g protein) } \\
\hline & Control & $\mathrm{pBI} 121$ & $\mathrm{pBI} 12$ PPAL & $\mathrm{pBI} 12 \mathrm{PPAL}$ \\
& & \multicolumn{4}{c}{$\begin{array}{c}\mathrm{I} \\
\text { transformant }\end{array}$} & $\begin{array}{c}\text { transformant } \\
\text { II }\end{array}$ \\
2 Days & $4.2 \pm 0.5$ & $4.4 \pm 0.6$ & $13.2 \pm 0.5$ & $12.5 \pm 0.7$ \\
28 days & $7.2 \pm 1.5$ & $11.9 \pm 2.2$ & $18.2 \pm 0.7$ & $17.1 \pm 0.9$ \\
\hline \multicolumn{5}{c}{ Growth (g fresh weight/flask) } \\
\hline 28 Days & $11.5 \pm 1.8$ & $12.8 \pm 1.0$ & $3.3 \pm 0.8$ & $3.0 \pm 0.6$ \\
\hline
\end{tabular}

formants. Of all the kanamycin resistant pBI12PPAL transformants selected, only two could be successfully cultured for analysis of growth and PAL activity (Table 1). One explanation for the slow growth is that the PAL gene had resulted in increased lignification or production of other phenylpropanoids. PAL activity was measured to determine the effect of the PAL transgene on enzyme activity and its relationship to root growth (Fig.5). PAL activity in pBI121 transformant I was initially much higher than in control roots without the PAL transgene, but the activity in control hairy roots soon increased, while that in pBI12PPAL transformant I decreased. At later stages of the experiment PAL activity increased in all hairy roots, but to a greater extent in pBI12PPAL transformant I such that their final activity was 1.5 times greater than controls. pBI12PPAL transformant II exhibited the same morphology, colour, growth rate and $\mathrm{PAL}$ activity (Data not shown). Although an increase in PAL activity of 1.5 times is not great, it may be sufficient to cause an increase in production of toxic phenolic compounds which could slow hairy root growth. Alternatively the in vivo PAL activity in $\mathrm{pBI} 12 \mathrm{PPAL}$ transformants could be relatively much higher than in control hairy roots if the parsley enzyme is not cor-

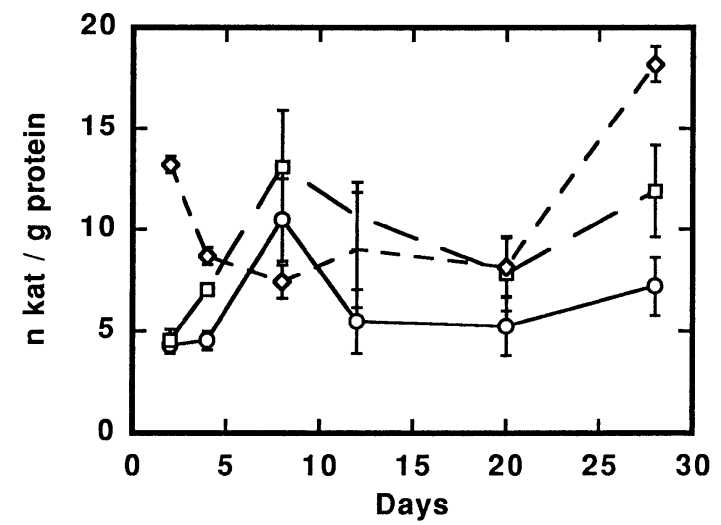

Fig. 5 Changes of the PAL activities in hairy roots transformed with pBI121 and pBI12PPAL during the culture period.

Values represent mean \pm s. e. m. from triplicate flasks. - $\bigcirc$ - Control hairy root, $-\square-$; pBI121 transformed hairy root, $-\diamond-$; pBI12PPAL transformed hairy root.

rectly regulated in Capsicum. These factors could explain the failure to obtain transformants with more than a 1.5 fold increase in PAL activity. These results are consistent with the proposal that PAL activity can influence root growth and phenylpropanoid production. Similar conclusions have been reached in two related studies. Introduction of a heterologous (Phaseolus) PAL gene into transgenic Nicotiana tabacum plants resulted in reduced PAL activity and interference with phenylpropanoid biosynthesis [15]. Transformation of tobacco cells using particle bombardment with a tobacco PAL cDNA, produced cells with four-fold higer PAL activity and two-fold higher content of scopoletin[16]. These results collectively show that genetic manipula tion of PAL activity can modify phenylpropanoid synthesis in plant cells, although some of the observations are not fully explained. Perhaps the introduction of a heterologous enzyme into plant cells disturbs the normal control systems which regulate PAL activity and the flux through the phenylpropanoid pathway. Whatever the explanation, such manipulation of PAL activity appears to offer real potential for the modification of phenylpropanoid production in plant cells. Furthermore, the use of disarmed Agrobacterium strains with hypocotyl explants should allow the regeneration of transgenic chilli pepper plants.

\section{Acknowledgments}

We wish to thank to Dr. K. Hahlbrock for providing parsley PAL-2 cDNA and to Dr. H. Kamada for providing $A$. rhizogenes strains.

This work was supported in part by Grant-in-Aid for Scientific Research on Priority Areas (05278212) from the Ministry of Education, Science and Culture of Japan. 
This work was performed in part using the facilities of the Biotechnology Research Center, The University of Tokyo.

\section{References}

[1] Lindsey, K., Yeoman, M. M., 1984. Planta, 162: 495-501.

[2] Lindsey, K., 1985. Planta, 165: 126-133.

[ 3 ] Hall, R. D., Yeoman, M. M., 1991. Planta, 185: 72 -80 .

[ 4 ] Sukrasno, N., Yeoman, M. M., 1993. Phytochem., 32: 839-844.

[5] Liu, W., Parrot, W. A., Hildebrand, D. F., Collins, G. B., Williams, E. G., 1990. Plant Cell Rep., 9: 360-364.

[6] Schenk, R. U., Hildebrandt, A. C., 1972. Can. J. Bot., 50: 199-204.

[7] Sekiguchi, S., Yamakawa, T., Kodama, T., Smith, S. M., Yeoman, M. M., 1996. Plant Tissue Culture Letters., 13: 219-221.

[ 8 ] An, G., 1987. Methods in Enzymol., 153: 292-305.

[9] Lois, R., Dietrich, A., Hahlbrock, K., Schulz, W.,
1989. EMBO J., 8: 1641-1648.

[10] Draper, J., Scott, R., Armitage, P., Walden, R., 1988. In "Plant genetic transformation and gene expression. A laboratory manual.”, p. 212-214, Blackwell Scientific Publications, Oxford.

[11] Hamill, J. D., Rounsley, S., Spencer, A., Todd, G., Rhodes, M. J. C., 1991. Plant Cell Rep., 10: 221224.

[12] Bolwell, G. P., Bell, J. N., Cramer, C. L., Schuch, W., Lamb, C. J., Dixon, R. A., 1985. Eur. J. Biochem., 149: 411-419.

[13] Bradford, M. M., 1976. Anal. Biochem., 72: 248252

[14] Jefferson, R. A., Kavanagh, T. A., Bevan, M. W., 1987. EMBO J., 6: 3901-3907.

[15] Elkind, Y., Edwards, R., Mavandad, M., Hedrick, S. A., Ribak, O., Dixon, R. A., Lamb, C. J., 1990. Proc. Natl. Acad. Sci. USA, 87: 9057-9061.

[16] Nagai, N., Yanagisawa, K., Mizuno, K., Imura, H., Taguchi, G., Shimosaka, M., Shibata, D., Okazaki, M., 1995. Plant Tissue Culture Lett., 12 : 165-171. 